\title{
Evaluation of renal vascular lesions using thrombomodulin and vascular cell adhesion molecule- 1 in patients with biopsy-proven lupus nephritis
}

\author{
Genhong Yao $\cdot$ Guodong Zhang $\cdot$ Lijun Ling
}

Received: 17 October 2008 /Revised: 11 February 2009 /Accepted: 11 March 2009

(C) Clinical Rheumatology 2009

The authors discovered post-publication that the data submitted was incorrect. According to the study data, the patients were all IV lupus nephritis patients. However, on further review, the authors found that some patients in the study were not IV lupus nephritis, which meant that the diagnosis was wrong and the clinical data and the levels of thrombomodulin and vascular cell adhesion molecule-1 were not correctly reported. The authors apologize for this error, and have requested the manuscript to be withdrawn.

The authors discovered post-publication that the data submitted was incorrect. According to the study data, the patients were all IV lupus nephritis patients. However, on further review, the authors found that some patients in the study were not IV lupus nephritis, which meant that the diagnosis was wrong and the clinical data and the levels of thrombomodulin and vascular cell adhesion molecule-1 were not correctly reported. The authors apologize for this error, and have requested the manuscript to be withdrawn.

G. Yao $(\bowtie)$

Department of Transfusion, Jinling Hospital,

Nanjing University Clinical School of Medicine,

Nanjing 210002, People's Republic of China

e-mail: yaogenhong@yahoo.com

G. Zhang

Nanjing Medical University,

Nanjing 210029, People's Republic of China

L. Ling

Nanjing University,

Nanjing 210093, People's Republic of China 